\title{
EVOLUTION OF COOLING-CHANNEL PROPERTIES FOR VARYING ASPECT RATIO
}

\author{
M. Pizzarelli, F. Nasuti, and M. Onofri \\ Sapienza University of Rome \\ Department of Mechanical and Aerospace Engineering \\ 18 Via Eudossiana, Rome I-00184, Italy
}

\begin{abstract}
A trade-off analysis is performed on a cooling channel system representative of liquid rocket engine cooling systems. This analysis requires multiple cooling channel flow calculations which are performed by means of a proper numerical approach, referred to as quasi-two-dimensional (2D) model. This model, which is suited to high-aspect-ratio cooling channels (HARCC), permits to have a fast prediction of both the coolant flow evolution and the temperature distribution along the whole cooling channel structure. Before using the quasi-2D model for the trade-off analysis, its validation by comparison with computational fluid dynamics (CFD) results is presented and discussed. The results show that the pump power required to overcome losses in the cooling circuit can be minimized selecting a channel shaped with a suitably high aspect ratio.
\end{abstract}

\section{INTRODUCTION}

Cooling channels with high aspect ratio of the cross section (channel height-towidth ratio, $\lambda=h / b$ ), often referred to as HARCC, have the potential to reduce the thermal strain in the wall material and to increase the material strength by substantially reducing its maximum temperature (Fig. 1). For this reason, HARCC have been studied by NASA since many years [1-4] and introduced in engines like the European Vulcain [5]. In fact, reducing wall temperature allows increasing engine life and cost of fabrication, as resulted during development and production of Space Shuttle main engine. However, it has been demonstrated [6] that if the channel aspect ratio is too high, the cooling efficiency vanishes. In fact, in case of strongly asymmetric distributed heat fluxes around the channel perimeter and cooling geometries with high aspect ratios, limited coolant mixing and inhomogeneous temperature distribution (also referred to as "thermal stratification") are expected. This, in turn, can lead to cooling inefficiency of HARCC. 


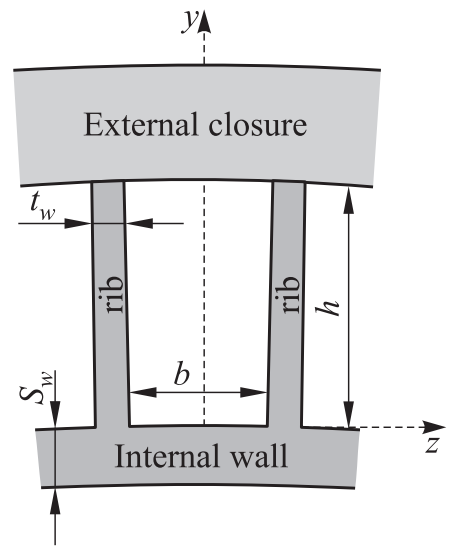

Figure 1 Schematic of cooling channel geometry

The aim of the present study is to emphasize that the optimum aspect ratio exists and depends on the set of assigned constraints. In particular, the constraints that characterize the cooling system are divided in two families: those related to the realization of the device (weight, dimensions, manufacturing capability, etc.) and those related to the system operation (maximum allowable wall temperature and coolant pressure drop, available coolant mass flow rate, coolant power loss, etc.). The present tradeoff analysis is performed on cooling channel conditions representative of those occurring in liquid rocket engines. In this study, the cooling channel aspect ratio that would minimize the coolant power loss is found with the constraint of fixed weight and channel height. The trade-off analysis is performed by means of a numerical approach presented and validated in [7-9]. This approach, which is based on a quasi-2D model, permits to have fast and complete information on the thermal evolution in cooling systems characterized by HARCC because of its capability to describe the thermal stratification as well as the temperature distribution along the whole cooling channel structure.

\section{VALIDATION OF THE MODEL}

In this section, a typical numerical solution obtained by means of the quasi$2 \mathrm{D}$ solver is compared to that obtained with a three-dimensional (3D) CFD solver [10] in order to give confidence to the quasi-2D solutions similar to those analyzed in the present study. The validity of the selected 3D CFD solver has been, in turn, proved in past studies by successful comparisons against experimental data relevant to cooling channel flow and heat transfer [10,11]. The channel geometry has been selected as equal to that of the European Vulcain engine in the throat region [5]. The channel cross section has an aspect ratio of $\lambda=8.46$ and the following dimensions (see Fig. 1): $h=11 \mathrm{~mm}, b=1.3 \mathrm{~mm}, t_{w}=1 \mathrm{~mm}$, and $s_{w}=1 \mathrm{~mm}$; the solid material is a CuAgZr copper alloy [12] whose thermal conductivity over the range of operative temperature is $k=295 \mathrm{~W} /(\mathrm{m} \cdot \mathrm{K})[13]$. Moreover, a straight portion of length $L=40 \mathrm{~cm}$ is considered and the channel surface is considered smooth. The mass flow rate and the inlet conditions of the hydrogen flow and the hot-gas-side wall temperature are selected accord- 
ing to Vulcain engine throat section data $[5,14]: \dot{m}=92.8 \mathrm{~g} / \mathrm{s}, T_{\mathrm{in}}=75 \mathrm{~K}$, $p_{\text {in }}=130 \mathrm{bar}$, and $T_{w, \mathrm{hg}}=800 \mathrm{~K}$. Hydrogen thermodynamic behavior is described by means of the modified Benedict-WebbRubin equation of state [15] for both solvers. The 3D CFD solution has been verified by a grid convergence analysis that has been presented in [16]. In particular, this analysis has shown that the numerical error can be neglected if compared with the difference between the 3D CFD and the quasi-2D solutions. The coolant domain computational mesh used for the $3 \mathrm{D}$ CFD simulations presented here is composed of $32 \times 128 \times 64$ volumes along the half-base, the height, and the axial direction of the channel, respectively. Taking advantage of the flow symmetry with respect to the vertical mid-plane, only onehalf of the physical domain is discretized. The volumes are clustered near the walls to accurately describe the turbulent boundary layer (the nondimensional distance $y^{+}$at the wall-adjacent cell is of order 1) while the axial distribution is uniform. The computational grid of the solid domain is composed of $640 \times 64$ volumes along the cross section and the axial direction of the channel, respectively; moreover, in each coordinate direction, the mesh distribution is uniform. Also, in this case, only one-half of the physical domain is discretized. Computational mesh of the channel cross section is presented in Fig. 2.

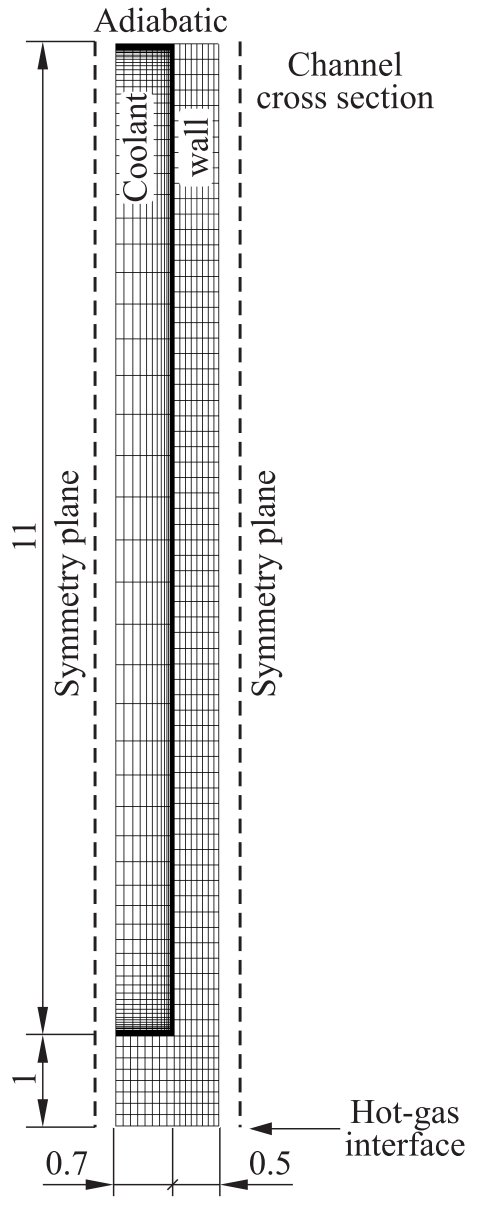

Figure 2 Computational meshes and boundary conditions of the 3D CFD simulation. Dimensions are in millimeters

The thermal behavior of the analyzed cooling channel is shown by temperature fields of both coolant and rib in Fig. 3, where the evolution of temperature is shown at inlet, half-length, and exit cross sections. Both 3D CFD and quasi-2D results are shown; in particular, the quasi-2D solution has been "extruded" in the channel-base direction for a better comparison with the 3D CFD solution. Looking first at the more accurate 3D CFD solution, it is possible to notice that the high-temperature flow region grows as the fluid moves from the inlet to the exit section, but it remains close to the hot wall along the whole length. As 


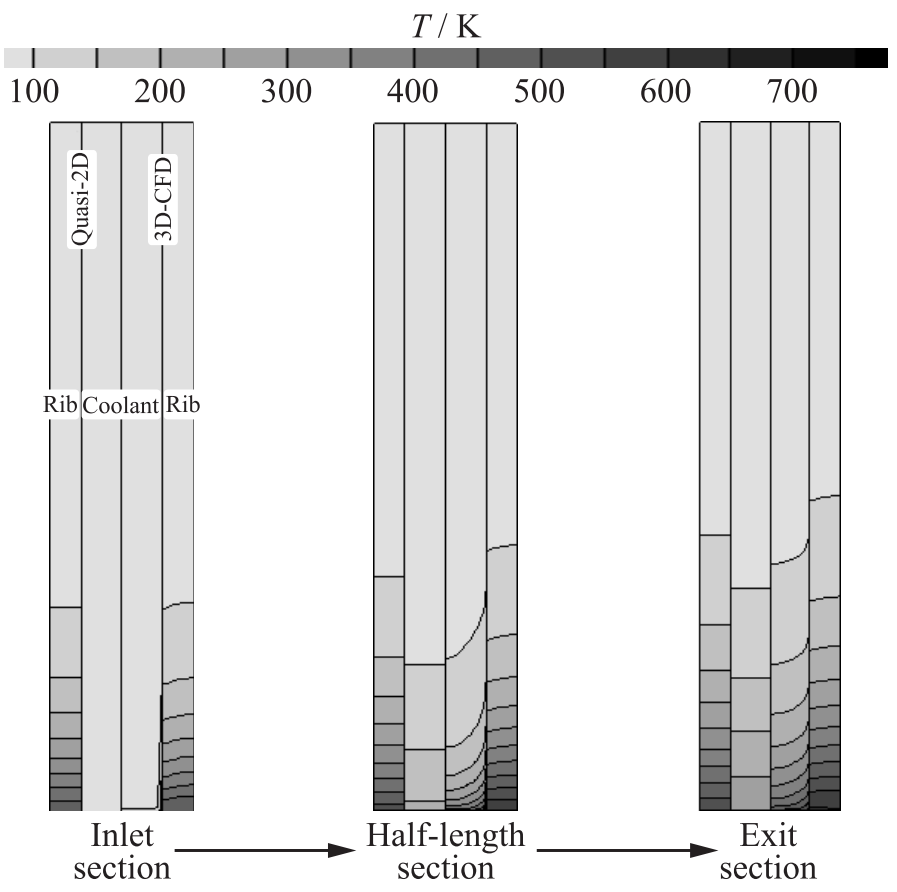

Figure 3 Coolant and rib temperature distributions at different cross sections, evaluated with both $3 \mathrm{D}$ CFD and quasi-2D models

a consequence, the fluid far from the bottom wall is nearly at the inlet condition and an evident nonuniform thermal distribution occurs. Figure 3 permits also to discuss the temperature spatial evolution in the high-conductivity solid material. As for the coolant-flow evolution, the average wall temperature increases with the channel length even if the hot-gas side wall temperature is enforced as a boundary condition at the hot-gas interface (see Fig. 2). In particular, the wall temperature keeps growing along the channel length. This is because in the upper part of the channel the wall temperature is nearly constant and its value is that of hydrogen at the inlet section (i. e., $T_{\mathrm{in}}=75 \mathrm{~K}$ ) and, therefore, temperature stratification of the solid material can further evolve. Comparing now the solutions of the two models, it is possible to notice that, despite the lack of information in the channel-base direction, the quasi-2D model is fairly able to reproduce both the fluid and the wall thermal stratification.

The quantitative comparison between the $3 \mathrm{D}$ CFD and the quasi-2D results in the radial direction is presented in Fig. $4 a$, where the coolant and wall temperature, and the heat flux profile along the radial direction and at the channel exit are displayed. In particular, the coolant temperature evaluated with the 


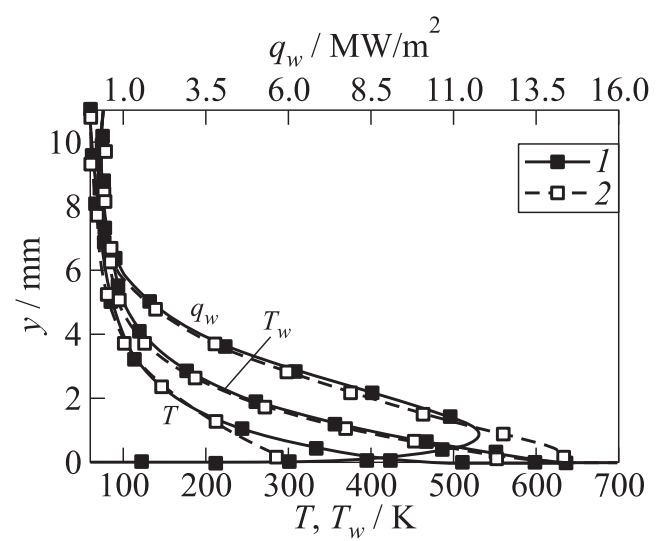

(a)

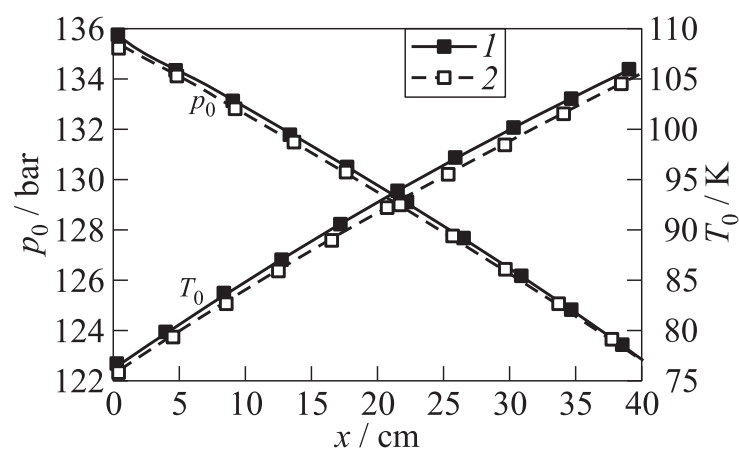

(b)

Figure 4 Comparison between 3D CFD (1) and quasi-2D (2) solutions: (a) radial distribution of coolant and wall temperature and heat flux at the last channel section; and $(b)$ coolant total pressure and bulk temperature

3D CFD model is that at the channel center plane. Note that the temperature profiles are characterized by an evident stratification, especially in the lower part of the channel where the fluid element is closer to the channel base, where temperature is higher. The comparison shows that wall temperature is very well reproduced by the simplified model, the maximum discrepancy being below $4 \%$. As for the coolant temperature and heat flux, the agreement is good only sufficiently far from the channel base; coolant temperature discrepancy is below $3 \%$ and heat transfer discrepancy is below $8 \%$ for $y>2 \mathrm{~mm}$. For radial sections below $2 \mathrm{~mm}$, the comparison must be performed with great care because the 3D CFD model describes the kinematic and thermal boundary layers while the simplified model does not. For that reason, the comparison of the 
two models in the near-wall region (that is, $y \simeq 0$ ) is not possible as already mentioned in [7]. The comparison between the 3D CFD and the quasi-2D results for the coolant total pressure and bulk temperature is presented in Fig. $4 b$. The definition of the bulk variables for the two different solutions is presented in details in [7]. Figure 4 shows that due to the incoming heat transfer rate from the hot-gas, the coolant temperature increases and that the effect of the incoming heat transfer and the wall friction results in a coolant pressure loss. The analysis of Fig. $4 b$ shows also that the behavior of the bulk variables obtained with the two different approaches is similar and the quasi-2D solution satisfactorily approximates the CFD solution: the coolant pressure drop of the two models differs by $3.6 \%$ and the temperature gain by $1.0 \%$. In particular, the quasi-2D solution underestimates both the pressure drop and the temperature gain. Also, local values of coolant total pressure and bulk temperature are slightly underestimated by the quasi- $2 \mathrm{D}$ model; maximum difference between the two solutions is $0.3 \%$ in case of pressure and $1.0 \%$ in case of bulk temperature. A better agreement between the two solutions could be reached by tuning of the semiempirical relations used in the quasi-2D model; in fact, despite the rectangular cross section of present channels, plain coolant skin friction and heat transfer relationships proposed for circular cross-section tubes have been used. However, as already demonstrated in [7], the overall good agreement of the quasi-2D solution with the reference 3D CFD solution in the case of HARCC does not justify further calibration of existing semiempirical models.

\section{TEST CASE DESCRIPTION}

The considered cooling system is composed of straight rectangular passages which contour a cylindrical chamber with an internal radius $r=150 \mathrm{~mm}$, a length $L=400 \mathrm{~mm}$, and an internal wall thickness $s_{w}=1 \mathrm{~mm}$. The reference cooling channel geometry is characterized by rib and channel thickness $t_{w}=b=2 \mathrm{~mm}$, and height $h=16 \mathrm{~mm}$, which corresponds to $\lambda=8$. The resulting number of cooling channels is $N=236$. Of course, for a given internal radius, the circumferential length of the cooling circuit, $2 \pi r=N\left(b+t_{w}\right)$, is constant for each channel geometry. The selected criterion to vary the channel aspect ratio is obtained considering the constraints of $h=$ const (which means the same overall dimensions) and $N h t_{w}=$ const (which means, for a given material, the same weight). The dependency of the geometric parameters on channel aspect ratio $\lambda$ is shown in Fig. 5. An important feature of the selected criterion is that the total coolant flow area $A_{\text {tot }}=N b h$ is constant. Note also that the reduction of the hydraulic diameter with $\lambda$ (see Fig. 5) guarantees that the cooling efficiency increases with the aspect ratio as discussed in $[8,16]$. 


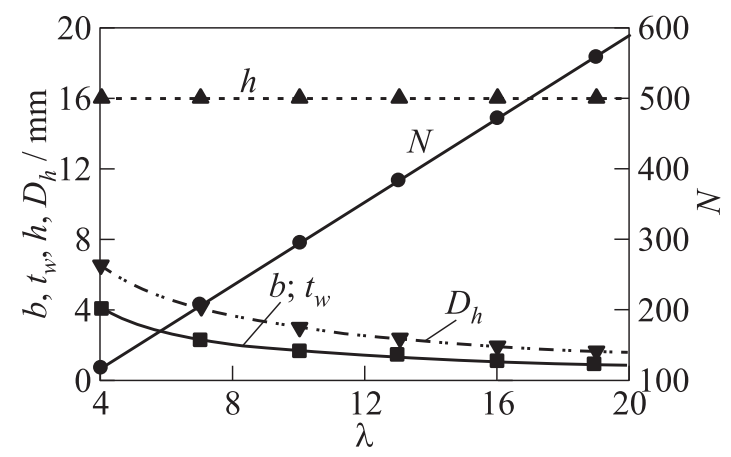

Figure 5 Dependency of the cooling circuit geometric parameters on channel aspect ratio

In this analysis, the solid material is assumed to have a hydrodynamically smooth surface and a thermal conductivity $k_{w}=390 \mathrm{~W} /(\mathrm{m} \cdot \mathrm{K})$ that is a typical value of the copper alloys used in rocket engine applications, such as the AMZIRC copper alloy [13]. The hot gas is characterized by a constant value of heat transfer coefficient and adiabatic wall temperature along the chamber length: $h_{w, \mathrm{hg}}=16000 \mathrm{~W} /\left(\mathrm{m}^{2} \mathrm{~K}\right)$ and $T_{\mathrm{aw}, \mathrm{hg}}=3500 \mathrm{~K}$, which are representative of the oparative conditions of high-performance rocket engines thrust chambers. The external wall is assumed adiabatic. The coolant is supercritical hydrogen and the inlet coolant conditions are $T_{\text {in }}=80 \mathrm{~K}$ and $p_{\text {in }}=80$ bar. Finally, for the reference case of $\lambda=8$, the mass flow rate of the single channel is $\dot{m}=0.171 \mathrm{~kg} / \mathrm{s}$; thus, the resulting Reynolds number of the turbulent flow is $\operatorname{Re}=4 \cdot 10^{6}$.

\section{RESULTS}

With reference to the cooling channel design of Fig. 5, two different parametric studies with variable channel aspect ratio are performed. The first parametric study refers to the case of constant coolant mass flow rate $\dot{m}_{\text {tot }}=40.28 \mathrm{~kg} / \mathrm{s}$, which is the value used for the reference case of $\lambda=8$. Also, inlet coolant temperature and pressure are the same for all the considered channel aspect ratios. The aspect ratio is varied from 4 to 20 . The resulting wall temperature and the heat flux at the hot-gas side, obtained with the quasi-2D model, are presented in Fig. 6.

The wall temperature decreases and the heat flux increases for increasing channel aspect ratio. Moreover, it is worth emphasizing a peculiar thermal behavior for increasing channel aspect ratio. In fact, if $\lambda \leq 8$, the wall temperature, after the flow development close to the channel inlet, reaches a maximum be- 


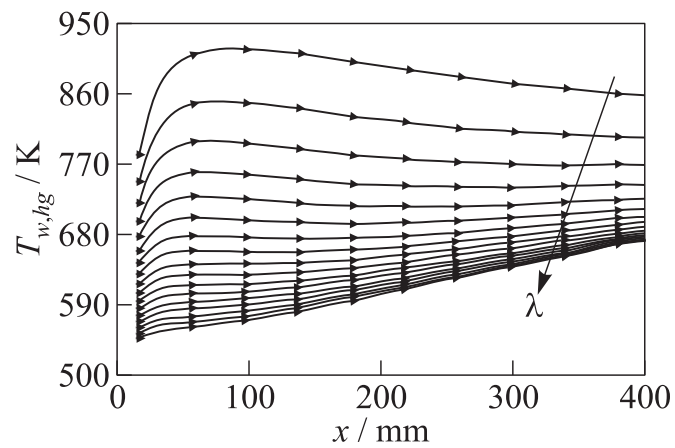

(a)

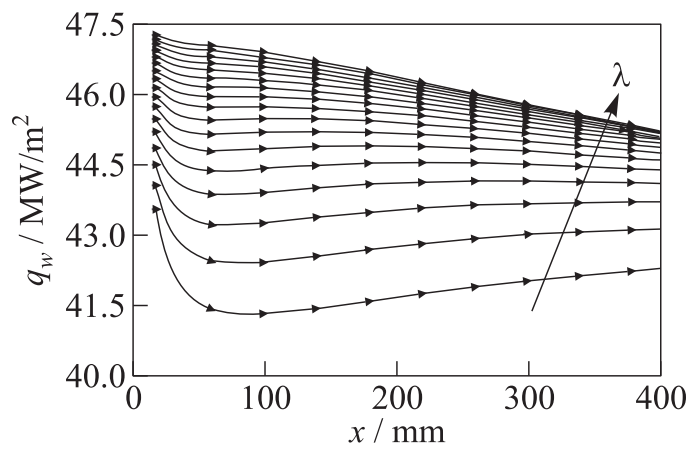

(b)

Figure 6 Hot-gas side parametric behavior; $\dot{m}_{\text {tot }}=40.28 \mathrm{~kg} / \mathrm{s} ; \lambda=4$ to 20 : (a) wall temperature and $(b)$ wall heat flux

tween $x \sim 50$ and $\sim 100 \mathrm{~mm}$ and then decreases (Fig. 6a). This is explained by the heat transfer decrease in the flow development region and the subsequent heat transfer increase due to the increasing coolant thermal conductivity with temperature; the minimum heat transfer coefficient reached after flow development causes the maximum wall temperature. The decrease is nearly $60 \mathrm{~K}$ in case of $\lambda=4$ while, in the reference case of $\lambda=8$, the wall temperature is nearly constant all along the channel length for $x \gtrsim 50 \mathrm{~mm}$. Further increase of the aspect ratio implies that the wall temperature progressively increases in the streamwise direction. The temperature increase is nearly $125 \mathrm{~K}$ in case of $\lambda=20$. Note that the wall temperature increase is limited by the channel length; that is, the longer the channel the larger the temperature increase. Thus, even if the wall temperature level of $\lambda=20$ is well below that of $\lambda=8$, it must be considered that a marked increase of the wall temperature is not desired in the actual application because of the possible excess of the maximum allowable 


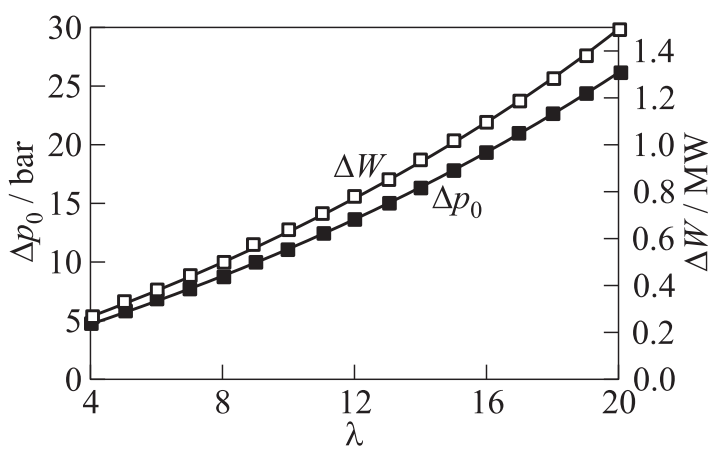

Figure 7 Cooling channel parametric behavior in case of imposed coolant mass flow rate; $\dot{m}_{\text {tot }}=40.28 \mathrm{~kg} / \mathrm{s}$

wall temperature after a certain channel length. The deterioration of the cooling capability in case of very high aspect ratio can be easily related to the heat flux decrease along the channel length (Fig. 6b). This effect is due to the limited mixing and thermal stratification within the coolant that takes place in case of high aspect ratio and has been already noticed in $[6,17]$.

In Fig. 7 , the coolant pressure drop, $\Delta p_{0}$, and the power loss, $\Delta W$ $=\dot{m}_{\text {tot }} \Delta p_{0} / \rho_{\text {in }}$ (where $\rho_{\text {in }}$ is the coolant density at the inlet), that is the requested coolant pump power to overcome pressure loss in the cooling circuit, is shown for the considered aspect ratio range. The results highlight that in case of constant coolant mass flow rate, the reduction of maximum wall temperature with increase of channel aspect ratio is offset by the increase of both pressure drop and power loss. This effect is due to the fact that for a given coolant velocity and total cross section area (i. e., for a given $\dot{m}_{\text {tot }}$ as in the present case), the increase of the total wetted perimeter with the channel aspect ratio (i.e., the decrease of $D_{h}$ with $\lambda$, see Fig. 5) implies a larger pressure drop. In particular, passing from $\lambda=4$ to 20 , these variables increase by a factor of about 13 .

The second parametric study is performed considering variable coolant mass flow rate with the constraint of maximum hot-gas side wall temperature. The selected value is $T_{w, \mathrm{hg}}=730 \mathrm{~K}$ which pertains to the reference case of $\lambda=8$ and $\dot{m}_{\text {tot }}=40.28 \mathrm{~kg} / \mathrm{s}$. Searching for the required coolant mass flow rate is made iteratively so that the wall temperature satisfies the constraint $T_{w, \mathrm{hg}}^{\max }=730 \mathrm{~K}$. Of course, due to the effect of limited coolant mixing and thermal stratification in case of high aspect ratios seen in Fig. $6 a$, the maximum wall temperature is encountered in the initial part of the channel length if $\lambda<8$ or at the channel exit if $\lambda>8$.

The behavior of the coolant pressure drop, mass flow rate, and power loss is shown in Fig. $8 a$ as a function of channel aspect ratio within the range $5 \leq \lambda$ 

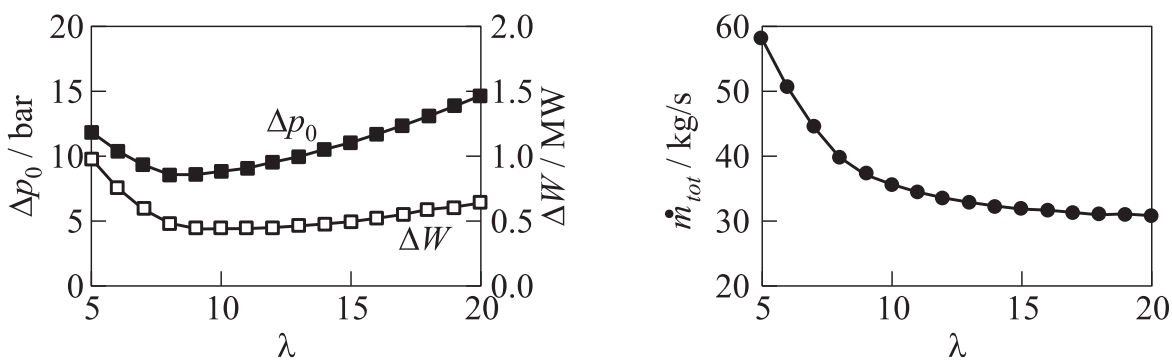

(a)
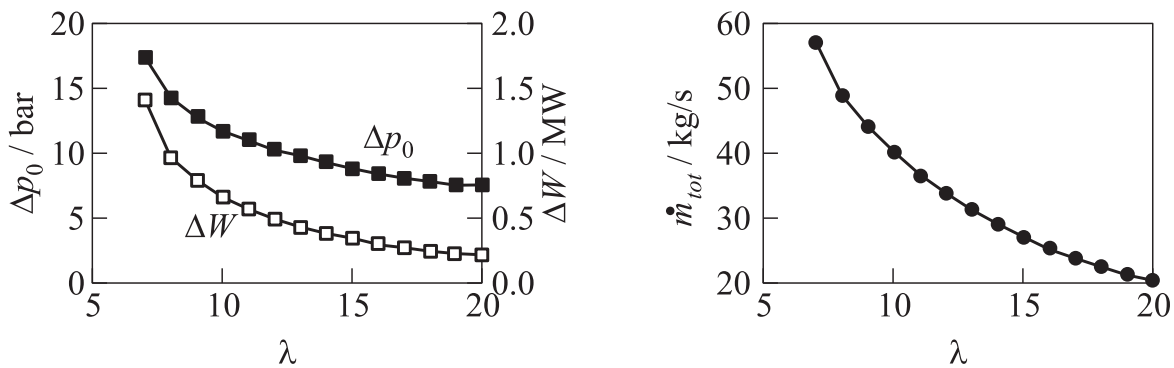

(b)

Figure 8 Cooling channel parametric behavior in case of imposed maximum wall temperature; $T_{w, \mathrm{hg}}^{\max }=730 \mathrm{~K}:(a)$ quasi-2D model; and $(b) 1 \mathrm{D}$ model

$\leq 20$. Note that the cases of aspect ratio lower than 5 are not considered because they are not able to satisfy the constraints of $T_{w, \mathrm{hg}}^{\max }=730 \mathrm{~K}$ and subsonic flow. The required constraint of imposed maximum wall temperature can be achieved with decreasing coolant mass flow rate as channel aspect ratio increases. In particular, passing from $\lambda=5$ to 20 , the mass flow rate almost halves, ranging from 58 to $31 \mathrm{~kg} / \mathrm{s}$. The coolant pressure drop exhibits a minimum for $\lambda=8$ which is $\Delta p_{0}=8.6$ bar; the pressure drop for smaller or larger aspect ratios is consistently higher, being $\Delta p_{0}=11.8$ bar for $\lambda=5$ and $\Delta p_{0}=14.6$ bar for $\lambda=20$. The minimum pressure drop is due to the fact that the coolant heat transfer coefficient increases with $\lambda$ because of the hydraulic diameter reduction but this behavior is limited by the "thermal stratification" that occurs for very high aspect ratios. These conflicting phenomena, for a given maximum wall temperature, lead to a minimum pressure drop. As a consequence, also the power loss through the cooling circuit presents a minimum value. This minimum is located around $\lambda=10$; with respect to $\lambda=5$ and 20, the power loss is reduced by $55 \%$ and $31 \%$, respectively.

Note that the minimum values of coolant pressure drop and power loss cannot be obtained by a one-dimensional (1D) model, because it is due to the limited 
mixing and the resulting thermal stratification within the coolant that takes place in case of high aspect ratio. This is demonstrated in Fig. $8 b$ where the same analysis shown in Fig. $8 a$ has been performed by means of a 1D model. The physical and mathematical modeling of the $1 \mathrm{D}$ approach is similar to that of the quasi-2D model but all the coolant variables vary only along the axial abscissa $x$ and, thus, no thermal stratification can be evaluated, as in any typical 1D cooling channel flow model. Moreover, the same semiempirical expressions of the quasi-2D model have been adopted for the $1 \mathrm{D}$ computations. The results of Fig. $8 b$ clearly demonstrate that if the coolant thermal stratification is not taken into account, as for the $1 \mathrm{D}$ approach, the minima of coolant pressure drop and power loss are not found. As a result, 1D modeling would lead to the wrong conclusion that increasing aspect ratio would always improve the cooling channel performance. The trade-off is due to stratification buildup that has to be properly modeled as done in the present study with the described quasi-2D model.

\section{CONCLUDING REMARKS}

A trade-off analysis has been carried out to study the performance of cooling channel for varying channel aspect ratio. Considering different aspect ratios and coolant mass flow rates, such that the wall temperature remains below a threshold value, an optimum value is finally identified as the channel configuration that yields the minimum power loss or the minimum pressure drop in the cooling circuit. Channel aspect ratio has been parametrically varied by assuming constant weight and height of the channels. This strategy has led to a minimum of power loss at $\lambda=10$. The minimum power is about half the value obtained at $\lambda=5$. In fact, the analysis has emphasized that increasing aspect ratio, the beneficial effect of HARCC reduces and for $\lambda>10$, it is canceled by the excessive coolant thermal stratification in the radial direction. The minimum of power loss can only be found if stratification is taken into account in the simulation. As an example, the results obtained by $1 \mathrm{D}$ modeling confirm that such simulations would lead to the wrong conclusion that increasing aspect ratio always improves the cooling channel performance.

\section{REFERENCES}

1. Quentmeyer, R. J. 1977. Experimental fatigue life investigation of cylindrical thrust chambers. AIAA Paper No. 1977-893.

2. Quentmeyer, R. J. 1990. Rocket combustion chamber life-enhancing design concepts. AIAA Paper No. 1990-2116. 
3. Carlile, J. A., and R. J. Quentmeyer. 1992. An experimental investigation of highaspect-ratio cooling passages. AIAA Paper No.1992-3154.

4. Wadel, M.F. 1997. Comparison of high aspect ratio cooling channel designs for a rocket combustion chamber. AIAA Paper No. 1997-2913.

5. LeBail, F., and M. Popp. 1993. Numerical analysis of high aspect ratio cooling passage flow and heat transfer. AIAA Paper No. 1993-1829.

6. Woschnak, A., D. Suslov, and M. Oschwald. 2003. Experimental and numerical investigations of thermal stratification effects. AIAA Paper No. 2003-4615.

7. Pizzarelli, M., S. Carapellese, and F. Nasuti. 2011. A quasi-2D model for the prediction of wall temperature of rocket engine cooling channels. Numer. Heat Tr. AAppl. 60:1-24.

8. Pizzarelli, M., F. Nasuti, and M. Onofri. 2013. Trade-off analysis of high-aspectratio-cooling-channels for rocket engines. Int. J. Heat Fluid Flow 44:458-467.

9. Betti, B., M. Pizzarelli, and F. Nasuti. 2014. Coupled heat transfer analysis in regeneratively cooled thrust chambers. J. Propul. Power 30:360-367.

10. Pizzarelli, M., F. Nasuti, R. Paciorri, and M. Onofri. 2009. Numerical analysis of three-dimensional flow at supercritical fluid in asymmetrically heated channels. AIAA J. 47:2534-2543.

11. Pizzarelli, M. 2013. Effectiveness of Spalart-Allmaras turbulence model in analysis of curved cooling channels. AIAA J. 51:2158-2167.

12. Schmidt, G., M. Popp, and Th. Fröhlich. 1998. Design studies for a 10 ton class high performance expander cycle engine. AIAA Paper No. 1998-3673.

13. Esposito, J. J., and R.F. Zabora. 1975. Thrust chamber life prediction. Vol. 1: Mechanical and physical properties of high performance rocket nozzle materials. NASA CR-134806. Final Report. Seattle, WA: Boeing Aerospace Co.

14. Fröhlich, A., M. Popp, G. Schmidt, and D. Thelemann. 1993. Heat transfer characteristic of $\mathrm{H}_{2} / \mathrm{O}_{2}$-combustion chambers. AIAA Paper No. 1993-1826.

15. Younglove, B. A. 1982. Thermophysical properties of fluids. I. Argon, ethylene, parahydrogen, nitrogen, nitrogen trifluoride, and oxygen. J. Phys. Chem. Ref. Data 11:1-353.

16. Pizzarelli, M., F. Nasuti, and M. Onofri. 2014. Effect of cooling channel aspect ratio on rocket thermal behavior. J. Thermophys. Heat Transfer 28:410-416.

17. Woschnak, A., and M. Oschwald. 2011. Thermo and fluidmechanical analysis of high aspect ratio cooling channels. AIAA Paper No. 2001-3404. 